\title{
Array-based comparative genomic hybridization in ulcerative colitis neoplasia: single non-dysplastic biopsies distinguish progressors from non-progressors
}

\author{
Mary P Bronner ${ }^{1}$, Marek Skacel ${ }^{2}$, David A Crispin ${ }^{3}$, Peter D Hoff ${ }^{4}$, Mary J Emond ${ }^{4}$, \\ Lisa A Lai ${ }^{3}$, Raymond R Tubbs ${ }^{1}$, Jacintha N O’Sullivan ${ }^{5}$, Peter S Rabinovitch ${ }^{6}$ and \\ Teresa A Brentnall ${ }^{3}$
}

\begin{abstract}
${ }^{1}$ Division of Pathology and Laboratory Medicine, Cleveland Clinic, Cleveland, OH, USA; ${ }^{2}$ Dahl-Chase Pathology Associates, Bangor, ME, USA; ${ }^{3}$ Department of Medicine, Division of Gastroenterology, University of Washington, Seattle, WA, USA; ${ }^{4}$ Department of Biostatistics, University of Washington, Seattle, WA, USA; ${ }^{5}$ St Vincent's University Hospital, Dublin, Ireland and ${ }^{6}$ Department of Pathology, University of Washington, Seattle, WA, USA
\end{abstract}

\begin{abstract}
Approximately $10 \%$ of ulcerative colitis patients develop colorectal neoplasia. At present, identification of this subset is markedly limited and necessitates lifelong colonoscopic surveillance for the entire ulcerative colitis population. Better risk markers are needed to focus surveillance onto the patients who are most likely to benefit. Using array-based comparative genomic hybridization, we analyzed single, non-dysplastic biopsies from three patient groups: ulcerative colitis progressors $(n=9)$ with cancer or high-grade dysplasia at a mean distance of $18 \mathrm{~cm}$ from the analyzed site; ulcerative colitis non-progressors $(n=8)$ without dysplasia during long-term surveillance; and non-ulcerative colitis normal controls $(n=2)$. Genomic DNA from fresh colonic epithelium purified from stroma was hybridized to 287 (low-density) and $\mathbf{4 3 4 2}$ (higher-density) feature bacterial artificial chromosome arrays. Sample-to-reference fluorescence ratios were calculated for individual chromosomal targets and globally across the genome. The low-density arrays yielded pronounced genomic gains and losses in 3 of $9(33 \%)$ ulcerative colitis progressors but in none of the 10 control patients. Identical DNA samples analyzed on the higher-density arrays, using a combination of global and individual high variance assessments, distinguished all nine progressors from all 10 controls. These data confirm that genomic alterations in ulcerative colitis progressors are widespread, even involving single non-dysplastic biopsies that are far distant from neoplasia. They therefore show promise toward eliminating full colonoscopic surveillance with extensive biopsy sampling in the majority of ulcerative colitis patients.
\end{abstract}

Modern Pathology (2010) 23, 1624-1633; doi:10.1038/modpathol.2010.161; published online 27 August 2010

Keywords: array comparative genomic hybridization; colonic neoplasia; ulcerative colitis

Long-term ulcerative colitis is characterized by chronic inflammatory injury to the colonic mucosa. A subset approximating $10 \%$ of these patients

Correspondence: Dr MP Bronner, MD, Department of Anatomic Pathology, Cleveland Clinic, L-25, 9500 Euclid Avenue, Cleveland, OH 44195, USA.

E-mail: bronnem@ccf.org

Received 16 April 2010; revised 28 June 2010; accepted 11 July 2010; published online 27 August 2010 develop cumulative genomic damage leading to colorectal neoplastic progression. ${ }^{-4}$ Currently, detection of early, curable neoplasia requires lifelong colonoscopic biopsy surveillance of the entire ulcerative colitis population, despite the knowledge that the great majority of these patients will never develop cancer. The large potential for sampling error is a major challenge. That the surface area of the average colon ranges between 0.5 to $1.0 \mathrm{~m}^{2}$ relative to the tiny size of a biopsy specimen (approximating $\mathbf{0 . 0 0 2 \%}$ of the colonic mucosa) and 
the fact that most neoplasias are not visible by routine colonoscopy combine to create the problem. Accordingly, a minimum of 33 jumbo, well-oriented random biopsy specimens must be examined histologically to detect dysplasia with even $90 \%$ confidence, ${ }^{5}$ a number that is rarely achieved in clinical practice. Chromoendoscopy and other new endoscopic imaging techniques show promise for improving the sampling error, ${ }^{6,7}$ but these technologies are not yet standard practice. Observer variability among pathologists and differentiation between inflammatory and neoplastic change are further considerable problems. Finally, at least half a million people in the United States have ulcerative colitis, for whom lifelong colonoscopic examination involves considerable discomfort, high expense, and time and effort for patients and physicians alike. Unquestionably, better risk markers than dysplasia are needed.

Greater understanding of the molecular alterations in ulcerative colitis neoplastic progression may facilitate discovery of improved cancer risk markers. Previous reports have identified large fields of genomic damage in neoplastic progression in ulcerative colitis. ${ }^{1-3,5,8-15}$ Of great clinical importance, recent data even suggest that the abnormal molecular field may involve the entire colon. This might be anticipated from the diffuse chronic inflammatory injury thought to drive the process. Importantly, the field includes mucosal samples lacking histological dysplasia that are far removed from morphologically neoplastic lesions. ${ }^{1-3,5,8-15}$ This offers great promise for reducing the serious problem of sampling error in surveillance. Such molecular markers could therefore focus individualized and more cost-effective surveillance onto the minority of ulcerative colitis patients who would benefit the most. Of at least equal importance, such markers would allow the majority of ulcerative colitis patients who never develop cancer to safely forego difficult and unnecessary surveillance procedures.

Although the potential of the genomic assays is exciting, they are technically difficult to perform. Furthermore, the distinction between ulcerative colitis progressors and non-progressors using these markers remains imperfect. None of the proposed molecular markers have yet been validated prospectively or developed into high-throughput tests amenable to clinical testing. To continue to explore and improve the discriminatory ability of genomic markers of cancer risk in ulcerative colitis, we now examine array-based comparative genomic hybridization. This technology allows a more comprehensive genome-wide scanning for chromosomal losses and gains over large numbers of targets in comparison with earlier techniques. ${ }^{16,17}$ Discrimination of the genomic 'signatures' in ulcerative colitis progressors relative to non-progressors may provide a more sensitive and specific biomarker of cancer risk.

\section{Materials and methods}

\section{Patients}

This research was approved by the Human Subject Division at the University of Washington and the Internal Review Board at the Cleveland Clinic. A total of 19 patients from the University of Washington were studied from three clinical groups: nine ulcerative colitis patients with cancer or high-grade dysplasia elsewhere in their colectomies (ulcerative colitis progressors), eight ulcerative colitis patients without dysplasia or flow-cytometric DNA ploidy abnormalities on extensive biopsy sampling while in long-term colonoscopic biopsy surveillance for a minimum of 10 years (ulcerative colitis non-progressors), and two non-ulcerative colitis control patients undergoing sigmoid colectomy for diverticulosis. Of the nine ulcerative colitis progressors, seven had adenocarcinoma and two had high-grade dysplasia as their most advanced neoplasia at colectomy. The colectomy specimens were extensively mapped histologically, by examining $>100$ sections per colon. For the non-progressors, an average of 44 jumbo, well-oriented biopsies was obtained at each ulcerative colitis surveillance colonoscopy. Colonoscopies were performed at 6month to 3-year intervals depending on prior surveillance findings. The average age of the 17 ulcerative colitis patients (eight men, nine women) was 45 years, and that of the two non-ulcerative colitis controls (both males) was 54 years. The known duration of ulcerative colitis was a mean of 17.7 years (range: 4-35 years) for ulcerative colitis progressors and 16.9 years (range: 10-25 years) for ulcerative colitis non-progressors. Table 1 indicates the clinical parameters relative to the extent of neoplastic progression and the known clinical cancer risk markers in the nine ulcerative colitis progressor patients.

\section{Biopsy Samples}

The samples of all progressors and two nonprogressors were obtained from densely mapped colectomy specimens. Those from the remaining six non-progressors were obtained from endoscopic biopsies from densely biopsied colonoscopies. All samples were rapidly frozen in cell culture minimal essential medium with $10 \%$ DMSO, as previously described. ${ }^{5}$ Tested colonic mucosal samples were split into half. One half was processed for comparative genomic hybridization as explained below. The other immediately adjacent half was routinely fixed, processed, and sectioned to determine the histological diagnoses, according to the consensus criteria of the Inflammatory Disease/Dysplasia Morphology Study Group. ${ }^{18}$ All diagnoses were confirmed by one pathologist (MPB). The single histologically nondysplastic biopsy samples were selected from within densely mapped regions of non-dysplastic 
Table 1 Extent of neoplastic progression in nine UC progressors

\begin{tabular}{|c|c|c|c|c|c|c|c|}
\hline $\begin{array}{l}\text { UC progressor } \\
\text { patient }\end{array}$ & $\begin{array}{l}\text { Most advanced } \\
\text { neoplastic } \\
\text { diagnosis }\end{array}$ & $\begin{array}{l}\text { Dukes' colorectal } \\
\text { carcinoma stage }\end{array}$ & $\begin{array}{l}\text { Incident neoplasia vs } \\
\text { prevalent neoplasia } \\
\text { vs unknown status }\end{array}$ & $\begin{array}{l}\text { Extent of } \\
\text { Colonic } \\
\text { disease }\end{array}$ & $\begin{array}{l}\text { Age of UC } \\
\text { onset } \\
\text { (years) }\end{array}$ & $\begin{array}{l}\text { UC disease } \\
\text { duration } \\
\text { (years) }\end{array}$ & $P S C$ \\
\hline $\mathrm{P} 1$ & Carcinoma & Dukes' B & Incident & Rectum & 61 & 13 & No \\
\hline P2 & High-grade dysplasia & - & Incident & Pancolitis & 7 & 35 & No \\
\hline P3 & Carcinoma & Dukes' C & Prevalent & Pancolitis & 20 & 13 & No \\
\hline $\mathrm{P} 4$ & Carcinoma & Dukes' B & Unknown & Pancolitis & 5 & 35 & No \\
\hline P5 & Carcinoma & Dukes' A & Prevalent & Pancolitis & 27 & 4 & No \\
\hline P6 & High-grade dysplasia & - & Prevalent & Pancolitis & 28 & 29 & Yes \\
\hline P7 & Carcinoma & Dukes’ B & Incident & Pancolitis & ? & Long $\mathrm{Hx}$ & No \\
\hline P8 & Carcinoma & Dukes' A & Incident & Pancolitis & 12 & 21 & No \\
\hline P9 & Carcinoma & Dukes' C & Unknown & Unknown & 38 & 13 & Yes \\
\hline
\end{tabular}

Hx, history; ?, unknown; UC, ulcerative colitis; PSC, primary sclerosing cholangitis; incident neoplasia, diagnosed while under endoscopic biopsy surveillance; prevalent neoplasia, diagnosed at initial endoscopy.

mucosa from the ulcerative colitis progressors. They were located at a mean distance of $18 \mathrm{~cm}$ (range: 2-79 cm; standard deviation (s.d.): $23.9 \mathrm{~cm}$ ) from the most advanced neoplastic lesion (either carcinoma or high-grade dysplasia, respectively), and were located in the rectosigmoid colon $(n=3)$, transverse colon $(n=5)$, or right colon $(n=1)$. The single nondysplastic samples from the non-progressors were obtained from the left colon $(n=5)$, right colon $(n=1)$, and rectum $(n=2)$. Both non-ulcerative colitis normal control samples were obtained from the sigmoid colon.

\section{Epithelial Isolation and DNA Extraction}

Epithelial crypt isolation was performed using an ethylenediaminetetraacetic acid shake-off technique to isolate $>90 \%$ pure colonic epithelial cells from mucosal stroma, as previously described. ${ }^{2}$ Whole genomic DNA was extracted using Gentra Puregene kit according to the manufacturer's instructions.

\section{Low-Density Arrays}

A commercially available genomic DNA microarray kit was utilized (GenoSensor Array 300; Vysis, Downers Grove, IL, USA), containing 287 oncologically representative genomic DNA targets from P1, PAC, and bacterial artificial chromosome libraries. Aliquots of sample and normal pooled reference DNA were DNase digested to generate 50 - to 800base pair (bp) fragments, labeled by random priming reaction (Random Priming Reaction kit; Vysis) with Cy 3-dCTP or Cy 5-dCTP (Perkin-Elmer, Boston, MA, USA), respectively, and hybridized in microarray hybridization buffer (Vysis) containing Cot-1 DNA at $80^{\circ} \mathrm{C}$ for $10 \mathrm{~min}$. Hybridization to the array slide at $37^{\circ} \mathrm{C}$ for $60 \mathrm{~h}$ in a humidified coplin jar with $2 \mathrm{ml} 50 \%$ formamide and $2 \times$ standard saline citrate (SSC) was followed by washes and counterstaining with $20 \mu \mathrm{l}$ of $4^{\prime}, 6$-diamidinophenylindole dihydrochloride IV solution (Vysis). The data obtained from the low-density arrays were analyzed using the built-in software of the GenoSensor ${ }^{\mathrm{TM}}$ Reader system (Vysis). Based on the average mean fluorescence intensity across the array, this software calculates the correlation coefficients among the triplicate spots for each target and displays the fluorescence ratios between the sample and reference DNA when they fulfill all quality control criteria set by the manufacturer.

\section{Higher-Density Arrays}

DNA was digested with DpnII and applied to a MinElute Reaction Clean-Up column (Qiagen). Random amplification and labeling steps were performed according to the method of Lieb with modifications. ${ }^{19}$ Briefly, a primer (primer A; 5'-GTT TCCCAGTCACGATCNNNNNNNNN-3') containing a degenerate $3^{\prime}$ end and a specific $5^{\prime}$ end was randomly annealed to DNA templates and extended with a T7 polymerase (Sequenase Version 2.0; USB, Cleveland, OH, USA). Then, a Cy3- or Cy5-labeled primer (5'-GTTTCCCAGTCACGATC-3'; Operon, Alameda, CA, USA) specific to the $5^{\prime}$ end of primer A was used to PCR amplify the templates generated in the first reaction. PCR reactions were then applied to a QIAquick PCR Purification column (Qiagen), blocking agents added (50 $\mu \mathrm{g}$ human Cot-1 DNA and $100 \mu \mathrm{g}$ yeast tRNA; Invitrogen, Carlsbad, CA, USA), and concentrated with a Microcon 10 filter (Millipore, Bedford, MA, USA).

The bacterial artificial chromosome array was manufactured at the Fred Hutchinson Cancer Research Center array facility and comprised 4342 clones, ${ }^{20}$ which were spotted in triplicate. The clones are a subset of those described in the bacterial artificial chromosome Resource Consortium, ${ }^{21}$ augmented with clones containing genes relevant to tumorigenesis and clones isolated from single-copy regions near the end of most chromosome arms. ${ }^{22}$ The genomic inserts of the bacterial artificial chromosomes average $166 \mathrm{~kb}$. Some sequence has 
been determined from each clone (eg, end sequence read(s), PCR-confirmed sequence-tagged site (STS) or gene, or the clone's entire sequence), allowing each clone to be positioned (at the midpoint of each clone) precisely within the human draft sequence assembled as of April 2003 ${ }^{23}$ (http://genome.ucsc. $\mathrm{edu} / \mathrm{)}$. Clones that were hybridized by fluorescence in situ hybridization (FISH) to multiple chromosomal locations or to a cytogenetic position inconsistent with a position in the draft sequence assembly were excluded from analysis, yielding 4153 clones used in the final analysis. ${ }^{21}$ These clones are distributed across the genome at a median spacing of $411 \mathrm{~kb}$ when the repetitive regions around centromeres and on the short arms of acrocentric chromosomes are excluded (25th percentile, $161 \mathrm{~kb}$; 75th percentile, $869 \mathrm{~kb}$; mean, $675 \mathrm{~kb}$; three intervals exceed $5 \mathrm{MB}$ ). The locations of $94 \%$ of the 4153 clones have been verified by FISH. The clones used in our analyses and details describing the coordinates are available from http:// www.fhcrc.org/labs/ trask/arraycomparative genomic hybridization. The clones are available from CHORI (http://bacpac.chori.org/).

To construct the array, bacterial artificial chromosome DNA was extracted from 1.5-ml cultures using a modified Qiagen R.E.A.L. Prep96 plasmid kit (Qiagen). Purified bacterial artificial chromosome DNA was then amplified using ligation-mediated PCR $^{23}$ and cleaned using the Multiscreen-PCR filtration system (Millipore) on a Beckman Coulter Biomek FX automated liquid-handling system (Beckman Coulter, Fullerton, CA, USA). Purified products were transferred to 384-well plates, airdried, and then resuspended in $3 \times$ SSC. Each amplified/purified bacterial artificial chromosome was then mechanically spotted in triplicate onto poly-L-lysine-coated slides using a GeneMachines Omnigrid Arrayer (GeneMachines, San Carlos, CA, USA).

Tumor and reference-pooled normal DNA labeled with Cy5 and Cy3, respectively, were combined and briefly dried in a speedvac. The samples were then resuspended in the hybridization mix $(50 \%$ formamide, $10 \%$ dextran sulfate, $3 \times \mathrm{SSC}, 1.5 \%$ SDS). Immediately following resuspension, samples were heated to $70^{\circ} \mathrm{C}$ for $10 \mathrm{~min}$ followed by 1 -h incubation at $37^{\circ} \mathrm{C}$ to allow blocking of repetitive sequences by human Cot-1 DNA. After incubation, the sample was applied to the microarray under a coverslip and hybridized at $37^{\circ} \mathrm{C}$ for $18 \mathrm{~h}$. Following hybridization, the arrays were washed in $50 \%$ formamide, $2 \times \mathrm{SSC}, \mathrm{pH} 7.0$, for $20 \mathrm{~min}$ at $45^{\circ} \mathrm{C}$, followed by a 7 -min wash at room temperature in $0.1 \mathrm{M} \mathrm{NaPO}_{4}, \mathrm{pH}$ 8.0, 0.1\% NP40. After the washes, the arrays were dried by centrifugation at 600 r.p.m. for $5 \mathrm{~min}$.

The higher-density arrays were scanned using the GenePix 4000B scanner (Axon Instruments, Union City, CA, USA), and the fluorescence data were processed using GenePix 3.0 image analysis software (Axon Instruments). For each spot, $\log _{2}$ ratio $=\log _{2}(\mathrm{Cy} 5 / \mathrm{Cy} 3)$ was calculated, where Cy5 and Cy3 refer to the median foreground fluorescent signals of the tumor and reference DNA, respectively. The $\log _{2}$ ratios on each array were normalized and corrected for intensity-based location adjustment using a block-level loess algorithm. ${ }^{24}$

\section{Reference DNA}

Two control DNA pools were used for arraybased comparative genomic hybridization analysis. The male control and female control pools each contained DNA from 15 cytogenetically normal individuals. For procedural quality control, all analyses were performed as sex-mismatch hybridizations. This allowed determination of chromosome $\mathrm{X}$ and $\mathrm{Y}$ copy number as an internal reference standard.

\section{Statistical Analysis}

The low-density array data were analyzed with respect to the overall extent of genomic abnormalities per sample as identified by the built-in GenoSensor software. The frequencies of genomic gains and losses in progressors, non-progressors, and non-ulcerative colitis controls were compared using descriptive statistics. The higher-density array data were analyzed with respect to the overall extent of genomic aberrations, as well as with respect to specific abnormalities differing between progressors and non-progressors. Copy number alterations were independently verified using comparative genomic hybridization-Miner ${ }^{25}$ with a three-SNP moving window and false discovery rate set at 0.01. Copy number estimates were manually curated for events with an average $\log _{2}$ intensity ratio of $>0.26$ (gains) or $<-0.32$ (losses). As a measure of the overall amount of copy number aberration over the entire genome, the following procedure was performed: An absolute value of the $\log _{2}$ copy number ratio for each locus was calculated (normal ratio 1, normal $\log _{2}$ ratio 0 ). The mean of the most extreme $5 \%$ of these absolute values was defined to be the 'extreme deviation score' for each patient. The $5 \%$ value was chosen arbitrarily, analogous to the common assumption that the most extreme $5 \%$ of a measurement on a population is most likely to be representative of biological abnormalities. In order to identify specific aberrations discriminating ulcerative colitis progressors from non-progressors, we restricted our search to the subset of bacterial artificial chromosomes showing a significantly higher variance in response than the overall sample variance in response. This selection was independent of the progressor/non-progressor status of the cases. One-sided chi-square test of equal variance and Bonferroni correction for multiple comparisons were used in this analysis. The Wilcoxon rank-sum 
test was then used to compare the responses of progressors and non-progressors for each of the identified candidate bacterial artificial chromosome clones.

\section{Results}

\section{Low-Density Arrays}

Using the low-density arrays, marked genomic alteration detected at 16 or more of the 287 loci on the array was identified in three of nine $(33 \%)$ biopsies from ulcerative colitis progressors (mean: 18.3 altered loci; range: 17-19; s.d.: 1.2). Only rare genomic changes detected at $<5$ loci were observed in the remaining six ulcerative colitis progressors (mean: 2.5 loci; range: 0-4; s.d.: 1.4), as well as in all eight ulcerative colitis non-progressors (mean: 1.3 loci; range: 0-3; s.d.: 1.1) and both non-ulcerative colitis controls (mean: 1.8; range 0-5; s.d.: 1.9). The absence of alterations in any of the 10 controls suggests good specificity. However, since two-thirds of the ulcerative colitis progressors went undetected, the low-density array provided only limited sensitivity. Larger arrays were then studied to assess whether this might improve the sensitivity.

\section{Higher-Density Arrays}

Visual inspection of the 4153 effective element bacterial artificial chromosome arrays showed patterns suggestive of increased chromosomal gains and losses (Figure 1c) and interstitial deletions (Figure 1d) in ulcerative colitis progressors, as compared with normal colon controls (Figure 1a) or ulcerative colitis non-progressors (Figure 1b). To create a quantitative measure of global copy number aberration throughout the entire genome, an absolute value of the $\log _{2}$ copy number ratio for each bacterial artificial chromosome target on the array was calculated. Using the mean of the most extreme $5 \%$ of these absolute values, an 'extreme deviation score' was calculated for each patient. In addition, as another independent metric to the deviation score, s.d.'s of the results across all measurements were also calculated for each patient. Both analyses demonstrated increasing genomic 'noisiness' for the ulcerative colitis progressors compared with nonprogressors as demonstrated in Figure 2a and b, respectively. With the exception of two ulcerative colitis progressors (P1 and P2), who showed similar degrees of alteration as non-progressors, the remaining seven progressors (78\%) displayed consistently greater extreme deviation scores and s.d.'s than any of the non-progressors. Notably, three especially unstable progressors were identified using both approaches and are shown in the right upper end of both the curves (P7, P8 and P9). These three patients were also the only three detected as markedly unstable using the low-density arrays.

Furthermore, after obtaining a global 'signature' of genomic aberrations for each patient, we assessed whether the presence of aberrations at particular chromosomal locations could discriminate among the two patient groups. We were interested in identifying chromosomal targets or combinations of targets which all or most progressors have in common and are absent in the majority of nonprogressors. Individual target variance analysis
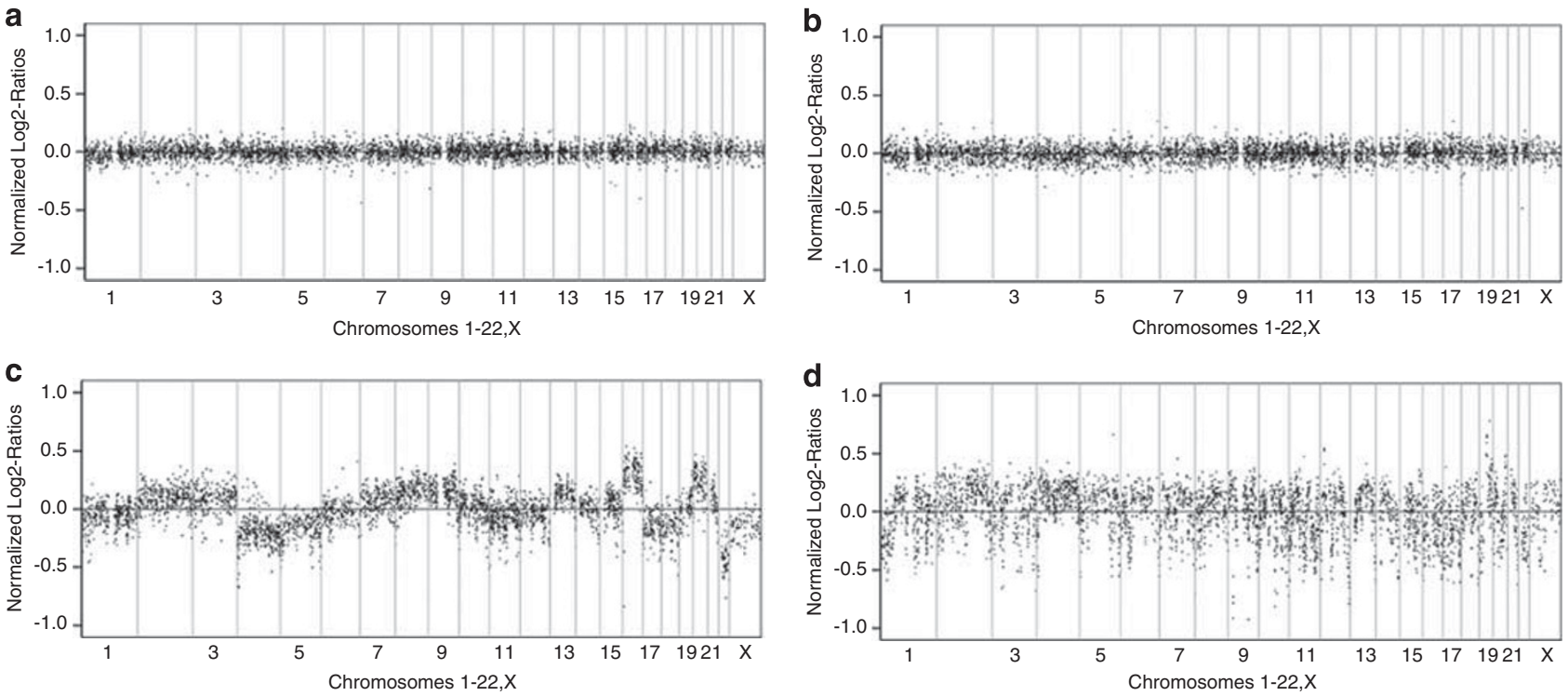

Figure 1 Higher-density bacterial artificial chromosome array whole genome $\log _{2}$ ratios plotted across all chromosomes consecutively from 1 through 22 plus the X chromosome. (a) Normal control non-ulcerative colitis patient 'C1'; (b) ulcerative colitis nonprogressor patient 'N1'; (c) ulcerative colitis progressor patient 'P8'; and (d) ulcerative colitis progressor patient 'P9'. These four representative patient results illustrate prominent chromosomal gains and losses (c) or interstitial deletions (d) in ulcerative colitis progressors in contrast to the minimal changes in non-progressor (b) and normal controls (a). 
confirmed the presence of a distinct pattern of genomic alteration in the ulcerative colitis progressors and showed an improved separation of these samples from non-progressors. Whereas the majority of aberrations detected genome-wide appeared random and did not discriminate between the patient groups, a smaller subset of changes showing the greatest variance were significantly more common in progressors. Using a one-sided chi-square test of equal variance with a Bonferroni-corrected significance level of $\alpha=0.05 / 4153$, a total of 40 candidate bacterial artificial chromosomal targets were identi-
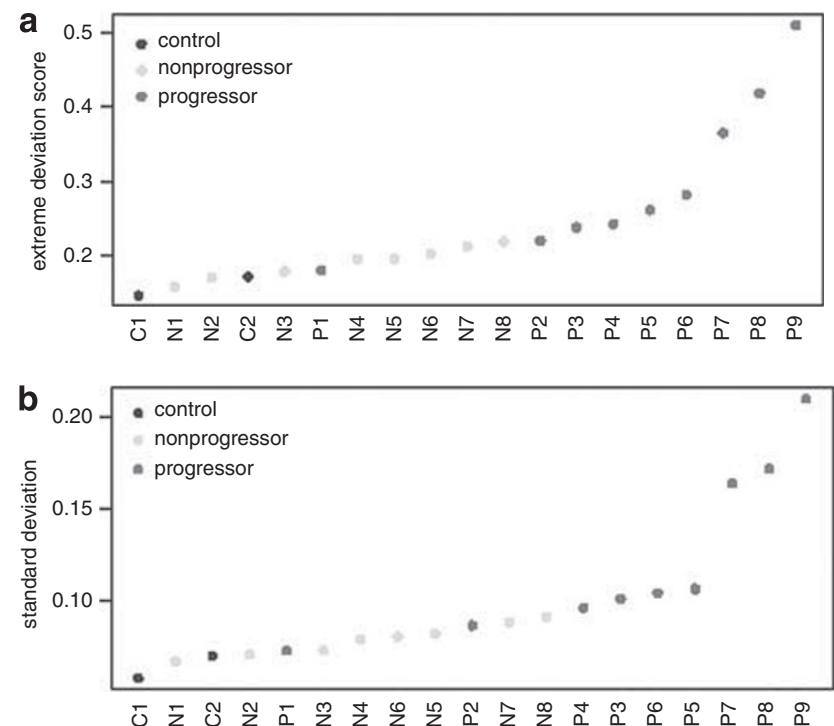

Figure 2 Extreme deviation score (a) and standard deviation (b) plots representing the absolute value of the $\log _{2}$ copy number ratio for the case-to-reference ratio at each locus (normal ratio 1, normal $\log _{2}$ ratio 0 ). The mean of the most extreme $5 \%$ of these absolute values was defined as the 'extreme deviation score' for each patient. The standard deviation examines standard deviation results across all measurements. Other than for progressors P1 and $\mathrm{P} 2$, the remaining $78 \%$ of progressors displayed greater genomic deviation relative to ulcerative colitis non-progressor and normal non-ulcerative colitis control patients. P, ulcerative colitis progressor; N, ulcerative colitis non-progressor; $\mathrm{C}$, nonulcerative colitis normal control patients. fied as having unusually high variance and were selected as candidate discriminators of progressors versus non-progressors. Wilcoxon's rank-sum tests and $t$-tests comparing the responses of progressors with those of non-progressors were performed for each candidate target separately. Eleven of the targets exhibited both $t$-statistics and rank-sum statistics that were significant at the nominal 0.05 level of significance, and are given in Table 2. All 11 of these targets demonstrated genomic loss in the ulcerative colitis progressors.

To provide results that are corrected for multiple testing, a Bonferroni correction was applied to maintain an overall false-positive error rate of 0.05 in declaring significant differences between progressors and non-progressors at particular chromosomal sites. With this method, a significant difference is declared only if the $P$-value is less than $0.05 / 40=0.00125$. This is the case for bacterial artificial chromosome targets 3905, 3906, and 3907 (RP11-7H17, RP11-196B3, and RP11-315M18), all of which map to the distal end of the long arm of chromosome 18 (18q23), as shown in Table 2. This clustering, which was independent of selection process, provides further confirmation of the statistical significance of these results. The differences for bacterial artificial chromosome 944 (RP11-460I19, maps to $4 p$ terminus) are of borderline significance by this criterion.

It should be noted that failure to show significance after correction for multiple testing for most of the 11 sites in Table 2 does not rule out the possibility that these sites have biological significance; it only means that we were unable to show significance after correction for multiple testing. This can result either from lack of biological significance or from lack of statistical power to identify differences with few patients and large numbers of possible predictors. The (normalized) log ratios among all of the patients for the three $18 \mathrm{q}$ sites, 3905, 3906, and 3907, are shown in Figure 3. As can be seen, $\log _{2}$ ratios from these three sites separate the progressors from the non-progressors

Table 2 Chromosomal sites with nominally significant differences (all losses) comparing UC progressors and non-progressors by array-based comparative genomic hybridization $(\alpha=0.05$ before correcting for multiple comparisons)

\begin{tabular}{|c|c|c|c|c|c|}
\hline$B A C^{\mathrm{a}}$ & $B A C I D^{\mathrm{a}}$ & Location & Std. dev. & $\mathrm{P}$-value (t-test) & P-value (rank sum) \\
\hline 944 & RP11-460I19 & 4p16 & 0.187 & 0.0015 & 0.001 \\
\hline 960 & RP11-101J14 & $4 \mathrm{p} 16$ & 0.203 & 0.0176 & 0.022 \\
\hline 1283 & RP11-94J21 & $5 p 15$ & 0.206 & 0.0131 & 0.0133 \\
\hline 1490 & RP11-2I16 & $5 q 35$ & 0.202 & 0.0158 & 0.035 \\
\hline 2594 & RP11-35C24 & $10 q 26$ & 0.239 & 0.0168 & 0.0279 \\
\hline 3476 & RP11-91J9 & $15 q 24$ & 0.19 & 0.0044 & 0.0101 \\
\hline 3796 & RP11-84J3 & $17 \mathrm{q} 25$ & 0.195 & 0.0074 & 0.0172 \\
\hline 3907 & RP11-315M18 & $18 q 23$ & 0.235 & $1 e-04$ & $6 e-04$ \\
\hline 3905 & RP11-7H17 & $18 \mathrm{q} 23$ & 0.196 & $1 e-04$ & $3 e-04$ \\
\hline 3906 & RP11-196B3 & $18 q 23$ & 0.238 & $6 e-04$ & $6 e-04$ \\
\hline 4299 & RP11-54I20 & $\mathrm{Xq} 28$ & 0.229 & 0.0138 & 0.0279 \\
\hline
\end{tabular}

${ }^{\mathrm{a}} \mathrm{BAC}$, bacterial artificial chromosome. 


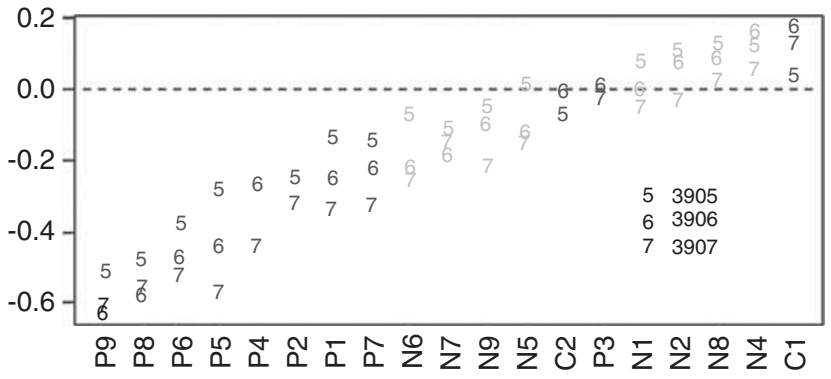

Figure 3 Ulcerative colitis progressor and non-progressor $\log _{2}$ ratios at the most informative bacterial artificial chromosomes 3905, 3906, and 3907.

except for one case (P3). Except for this one case, all the remaining progressors have negative values that are large when compared with the mean and s.d. of responses across all sites (mean -0.001, s.d. 0.11), suggesting chromosomal loss at the locations corresponding to these sites. In contrast, the distributions of the $\log _{2}$ ratios among the non-progressors are centered on zero and are relatively small in magnitude, suggesting that there is no relative chromosomal gain or loss. This suggests that progressors can generally be characterized by chromosomal loss on the distal tip of chromosome 18q.

\section{Discussion}

It is hypothesized that genomic alterations leading to ulcerative colitis neoplasia result from longstanding chronic inflammatory mucosal injury. However, only an estimated $10 \%$ minority of the ulcerative colitis population ever develops neoplasia. As such, improved markers to identify this atrisk group are greatly needed. This study identifies promising molecular markers using array-based comparative genomic hybridization.

Discoveries in the past several years have advanced our understanding of the molecular biology of ulcerative colitis. Widespread genomic alterations have been demonstrated in ulcerative colitis progressors with neoplasia, but not in non-progressors, using assays of telomere shortening and anaphase bridge development, ${ }^{2}$ DNA-fingerprinting by arbitrarily primed PCR,${ }^{13-15}$ preclonal chromosomal gains and losses by FISH, ${ }^{1,2,28}$ metaphase-comparative genomic hybridization, ${ }^{26-28}$ and estrogen receptor gene promoter hypermethylation. ${ }^{29}$

To our knowledge, this is the first report of arraybased comparative genomic hybridization in ulcerative colitis. Our aim was to interrogate the genome broadly to (1) identify specific alterations or patterns of chromosomal alterations that distinguish ulcerative colitis progressors from non-progressors, and (2) to better understand the early events that lead to neoplastic progression in ulcerative colitis by analyzing mucosa without dysplasia. Array technology permits high-resolution genomic scanning for chromosomal losses and gains over many thousands of genomic targets. The much higher resolution afforded by array technology is a major advance over the earlier comparative genomic hybridization technology using metaphase chromosomes. ${ }^{26-28}$ The higher-density 4298 feature BAC array used in this study has a mean intermarker spacing of $675 \mathrm{~kb}$ in comparison with the approximate $5-\mathrm{Mb}$ resolution of metaphase chromosomes. Metaphase-comparative genomic hybridization also necessitates considerable cytogenetic expertise for the morphological evaluation of hybridization signals along physical chromosomes rather than the automated signal detection of known chromosomal sites spotted onto systematic arrays. Regardless of the differences in these technologies, it is encouraging that a few differential chromosomal sites identified by earlier metaphase-comparative genomic hybridization were also observed in our array results, specifically chromosomal losses on 15, 5q, and 18q. ${ }^{26-28}$

An initial low-resolution array (287 clones) provided proof of principle that a greater degree of clonal genomic alteration was in fact present in nondysplastic mucosa from $33 \%$ of the progressors (three of nine) in contrast to none of the eight nonprogressors or two normal controls. The low-density array data additionally provided preliminary data regarding locations of highest-frequency genomic alterations. As anticipated, subsequent analysis of the identical DNA samples, using markedly higherdensity arrays (4153 clones), demonstrated this phenomenon on a much larger scale, distinguishing all nine progressors from all of the eight nonprogressors and two normal controls, when analyzed by a combination of statistical measures.

In addition, analysis of the higher-density arrays identified specific and unique genomic losses associated with neoplastic progression in dysplasia-free colonic samples from ulcerative colitis progressors. Of importance, the biopsy samples were obtained far distant from their respective tumors, highlighting the widespread nature of the genomic alterations within the colon. However, given the 4153 clones analyzed, it is not unlikely that due to chance alone a few targets in the progressors will all have gains or losses below some threshold and non-progressors above, or vice-versa, which could thus falsely discriminate perfectly among the groups. If the responses are not large in magnitude, then it is likely that the group differences are a result of experimental noise and are not due to consistent chromosomal gains or losses. To eliminate these from consideration, we restricted our search for potential discriminating targets to the subset of targets having a significantly higher variance in response than the overall sample variance in response. This selection was independent of the progressor/non-progressor status of the cases. The reasoning behind this selection criterion is as follows: for most genomic locations, most of the 19 cases do not have genomic abnormalities, and have array response intensity deviations that are relatively small in magnitude and are centered 
around 0 . In contrast, if many cases have abnormalities at a location corresponding to a given clone, then many of the 19 responses will be large in magnitude and the sample variance for that target will be large. By selecting for consideration only those bacterial artificial chromosomes with high variance, we are selecting targets for which there is evidence of genomic aberration across some of the 19 cases, although no consideration of group membership is taken into account, eliminating the bias.

Although a portion of the chromosomal gains and losses were seen in both progressors and nonprogressors, most of them were random and were not consistent between biopsies. However, a smaller subset of changes with great variance was nonrandom, as shown by $t$-tests comparing their distribution among progressors and non-progressors (Table 2 lists the 11 most significant). Such nonrandom losses of several genomic regions were observed in progressors, with the most powerful discriminator being a chromosomal loss on RP11196B3, which maps to the distal tip of the 18q arm (18q23). Two adjacent and overlapping targets at 18q were also highly discriminant. The 18q23 loss was detected in eight of nine (89\%) progressors and was not seen in any of the eight non-progressors or two normal controls. Other than several STSs, there is only one known gene in this area, named 'nuclear factor of activated T-cells, cytoplasmic, calcineurindependent 1' (NFATC1), according to the NCBI clone registry. Of interest, this transcription factor, initially identified as an important modulator of Tcell cytokine production, ${ }^{30,31}$ has been found to have increasing roles outside the immune system. A recent study by Duque and colleagues documents its importance in COX-2 regulation in colorectal carcinoma cell lines. ${ }^{32}$ COX-2 upregulation is welldescribed in colorectal tumorigenesis, both sporadic and ulcerative colitis forms. ${ }^{12}$

It is interesting that this $18 \mathrm{q}$ as well as other highly discriminant loci (5p15.33 and 10q26.3) determined by array-based comparative genomic hybridization are located in subtelomeric chromosomal regions. This finding further implicates a chromosomal bridge-fusion-breakage mechanism in relation to telomere shortening in ulcerative colitis neoplastic progression ${ }^{1,2}$ and in other cancers. $^{33,34}$ Subtelomeric regions may be inherently more prone to breakage after telomere attritionmediated end-to-end fusions with breakage at anaphase.

It is also noteworthy that the highest-variance non-random chromosomal alterations were all genomic losses (Table 2). This implicates loss of tumor suppressor genes as an important mechanism of the molecular pathogenesis of ulcerative colitis neoplasia.

These array-based comparative genomic hybridization data provide evidence that genomic changes associated with neoplastic progression in ulcerative colitis are sufficiently advanced to be clonal, which is intriguing, as they are present in non-dysplastic single random biopsy samples from ulcerative colitis progressors. That they are clonal derives from the knowledge that the detection threshold for comparative genomic hybridization gains or losses requires the majority of cells to demonstrate a given alteration. ${ }^{34}$

Three progressors failed to simultaneously demonstrate all three statistical measures of arraybased comparative genomic hybridization alteration. Specifically, one of the tested ulcerative colitis progressors (P3) in our small series of nine patients did not exhibit the 18q alterations (Figure 3), and an additional two ulcerative colitis progressors (P1 and P2) failed to demonstrate the random global alterations assessed by the extreme 5 th percentile deviation and s.d. analyses (Figure 2a and b). This could be related to the still limited resolution of $\sim 1 \mathrm{MB}$ afforded by even the higher-density 4298 bacterial artificial chromosome arrays utilized in this study. The improved sensitivity gained by the higher-density array supports this hypothesis. Improved resolution afforded by SNP, oligo and tiling, or submegabase-resolution tiling arrays may improve the sensitivity even further.

These three patients' results may also relate to sampling error within the colon, namely that more than one biopsy may be necessary to identify progressors and non-progressors. Future mapping studies of the distribution of CGH alterations in the colon of both progressors and non-progressors will be important.

A final potential explanation for why three ulcerative colitis progressors were not detected by all three statistical measures in this study could be that these patients differed clinically. This was true for the two progressors that lacked global genomic instability, namely P1 and P2 (Table 1). Progressor P2 had incident high-grade dysplasia that was detected during a prolonged 20 years of surveillance endoscopy, in comparison with the much more advanced prevalent adenocarcinomas in the majority of the remaining progressors. Further, progressor P1 was the only ulcerative colitis progressor without ulcerative pancolitis. Instead, progressor P1 had disease limited to the rectum rather than the pancolonic ulcerative colitis that was found in the remaining progressors. Numerous studies document that ulcerative proctitis appears to be a different disease with limited to no increased cancer risk. Surveillance is, in fact, not recommended for the ulcerative proctitis variant of ulcerative colitis. ${ }^{35-37,38}$ The biopsy sample analyzed by comparative genomic hybridization for patient P1 was derived from the unaffected sigmoid colon without changes in ulcerative colitis. It is possible that patient P1, who was also 74 years of age, actually developed a sporadic-type rectal cancer rather than an ulcerative colitis cancer. This remains speculation, but this patient was certainly clinically 
distinct from the remaining ulcerative colitis progressors. Thus, it is possible that these clinical differences may explain at least part of the variant array-based comparative genomic hybridization results for these three ulcerative colitis progressors that were not detected by all three statistical measures.

The fact that far-removed single biopsies lacking dysplasia in our small series of 17 ulcerative colitis cases were able to distinguish between progressors and non-progressors is remarkable. From a clinical perspective, this adds further credence to the concept that full colonoscopy with extensive biopsy sampling, which is currently the standard of care, could possibly be eliminated in the future. This follows from the finding that even single biopsies may harbor the genomic signature identifying ulcerative colitis progressors that could focus surveillance efforts onto the subset that is most likely to benefit. Natural history data on the development of genomic alterations during ulcerative colitis progression remain to be determined, as the current study only evaluated biopsies at the time of synchronous high-grade dysplasia or carcinoma. Longitudinal and prospective validation studies are underway.

In summary, array-based comparative genomic hybridization is a powerful discovery technique for genomic markers of ulcerative colitis cancer risk to better identify the minority subset of patients who are in need of intensive colonoscopic surveillance. By sequentially utilizing low- and high-resolution arrays, we demonstrate that the power of this approach increases with the increasing array target density. On comparing the patterns of genomic alteration in ulcerative colitis progressors with those of non-progressors, random and specific alterations identify progressors with $100 \%$ sensitivity and specificity in our small series of 17 ulcerative colitis patients. These results were achieved using only single non-dysplastic biopsies that were far distant from the patients' neoplasms. Of the changes identified, loss at the subtelomeric region of chromosome 18q appears to be the most powerful marker, along with several other subtelomeric losses, further implicating the telomere-shortening mechanism with bridge-breakage-fusion cycles previously identified in ulcerative colitis neoplastic progression. These array data represent an important additional step toward understanding of molecular events in neoplastic development in ulcerative colitis, and further demonstrate the great promise of molecular markers in even single biopsies to advance ulcerative colitis cancer surveillance.

\section{Acknowledgement}

This work was supported by NIH Grant R01 CA068124.

\section{Disclosure/conflict of interest}

The authors declare no conflict of interest.

\section{References}

1 O’Sullivan J, Bronner MP, Brentnall TA, et al. Chromosomal alteration is related to telomere shortening in a human preneoplastic disease (ulcerative colitis). Nat Genet 2002;32:280-284.

2 Bronner MP, O’Sullivan JN, Rabinovitch PS, et al. Genomic biomarkers on distant non-dysplastic biopsies distinguish UC progressors with neoplasia from dysplasia-free nonprogressors. Am J Pathol 2008;173: 1853-1860.

3 Brentnall TA, Rubin CE, Crispin DA, et al. A germline substitution in the MSH2 gene is associated with neoplasia in UC. Gastroenterology 1995;109:151-155.

4 Brentnall TA. Molecular underpinnings of cancer in UC. Curr Opin Gastroenterol 2003;19:64-68.

5 Rubin CE, Haggitt RC, Burmer GC, et al. DNA aneuploidy in colonic biopsies predicts future development of dysplasia in UC. Gastroenterology 1992;103: 1611-1620.

6 Rutter MD, Saunders BP, Schofield G, et al. Pancolonic indigo carmine dye spraying for the detection of dysplasia in UC. Gut 2004;53:256-260.

7 Kiesslich R, Neurath MF. Chromoendoscopy: an evolving standard in surveillance for UC. Inflamm Bowel Dis 2004;10:695-696.

8 Burmer GC, Rabinovitch PS, Haggitt RC, et al. Neoplastic progression in UC: histology, DNA content, and loss of a p53 allele. Gastroenterology 1992;103: 1602-1610.

9 Brentnall TA, Crispin DA, Rabinovitch PS, et al. Mutations in the p53 gene: an early marker of neoplastic progression in UC. Gastroenterology 1994;107:369-378.

10 Bronner MP, Culin C, Reed JC, et al. The bcl-2 protooncogene and the gastrointestinal epithelial tumor progression model. Am J Pathol 1995;146:20-26.

11 Issa JP, Ahuja N, Toyota M, et al. Accelerated agerelated $\mathrm{CpG}$ island methylation in UC. Cancer Res 2001;61:3573-3577.

12 Agoff SN, Brentnall TA, Crispin DA, et al. The role of COX-2 in UC-associated neoplasia. Am J Pathol 2000;157:737-745.

13 Chen R, Rabinovitch PS, Crispin DA, et al. The initiation of colon cancer in a chronic inflammatory setting. Carcinogenesis 2005;26:1513-1519.

14 Chen R, Bronner MP, Crispin DA, et al. Characterization of genomic instability in UC neoplasia leads to discovery of putative tumor suppressor regions. Cancer Genet Cytogenet 2005;162:99-106.

15 Chen R, Rabinovitch PS, Crispin DA, et al. DNA fingerprinting abnormalities can distinguish UC patients with dysplasia and cancer from those who are dysplasia/cancer-free. Am J Pathol 2003;162: $665-672$.

16 Solinas-Toldo S, Lampel S, Stilgenbauer S, et al. Matrix-based comparative genomic hybridization: biochips to screen for genomic imbalances. Genes Chromosomes Cancer 1997;20:399-407.

17 Pinkel D, Segraves R, Sudar D, et al. High resolution analysis of DNA copy number variation using com- 
parative genomic hybridization to microarrays. Nat Genet 1998;20:207-211.

18 Riddell RH, Goldman H, Ransohoff DF, et al. Dysplasia in inflammatory bowel disease: standardized classification with provisional clinical applications. Human Pathol 1983;14:931-968.

19 Lieb J, Liu X, Botstein D, et al. Promoter-specific binding of Rap1 revealed by genome-wide maps of protein-DNA association. [erratum appears in Nat Genet 29:100; 2001]. Nat Genet 2001;28:327-334.

20 Loo LW, Grove DI, Williams EM, et al. Array comparative genomic hybridization analysis of genomic alterations in breast cancer subtypes. Cancer Res 2004;64: 8541-8549.

21 Cheung V, Nowak N, Jang W, et al. Integration of cytogenetic landmarks into the draft sequence of the human genome. Nature 2001;409:953-958.

22 Knight SJ, Lese CM, Precht KS, et al. An optimized set of human telomere clones for studying telomere integrity and architecture. Am J Hum Genet 2000;67: 320-332.

23 Kent W, Sugnet C, Furey T, et al. The human genome browser at UCSC. Genome Res 2002;12:996-1006.

24 Yang YH, Dudoit S, Luu P, et al. Normalization for cDNA microarray: a robust composite method addressing single and multiple slide systematic variation. Nucleic Acids Res 2002;30:e15.

25 Wang P, Kim Y, Pollack J, et al. A method for calling gains and losses in array CGH data. Biostatistics 2005;6:45-58.

26 Kupka S, Schröder K, Porschen R, et al. Comparative genomic hybridization analysis of chromosomal alterations in patients with long-standing UC. Int J Oncol 2001;19:489-494.

27 Willenbucher RF, Aust DE, Chang CG, et al. Genomic instability is an early event during the progression pathway of ulcerative-colitis-related neoplasia. Am J Pathol 1999;154:1825-1830.

28 Willenbucher RF, Zelman SJ, Ferrell LD, et al. Chromosomal alterations in ulcerative colitis-related neoplastic progression. Gastroenterology 1997;113:791-801.
29 Fujii S, Tominaga K, Kitajima K, et al. Methylation of the oestrogen receptor gene in non-neoplastic epithelium as a marker of colorectal neoplasia risk in longstanding and extensive UC. Gut 2005;54: 1287-1292.

30 Serfling E, Berberich-Siebelt F, Chuvpilo S, et al. The role of NF-AT transcription factors in T cell activation and differentiation. Biochim Biophys Acta 2000; 1498:1-18.

31 Rao A, Luo C, Hogan PG. Transcription factors of the NFAT family: regulation and function. Annu Rev Immunol 1997;15:707-747.

32 Duque J, Fresno M, Iniguez MA. Expression and function of the nuclear factor of activated $\mathrm{T}$ cells in colon carcinoma cells: involvement in the regulation of cyclooxygenase-2. J Biol Chem 2005;280:8686-8693.

33 Soler D, Genesca A, Arnedo G, et al. Telomere dysfunction drives chromosomal instability in human mammary epithelial cells. Genes Chromosomes Cancer 2005;44:339-350.

34 Lim G, Karaskova J, Beheshti B, et al. An integrated Mband and submegabase resolution tiling set (SMRT) CGH array analysis of focal amplification, microdeletions, and ladder structures consistent with breakagefusion-bridge cycle events in osteosarcoma. Genes Chromosomes Cancer 2005;42:392-403.

35 Kallioniemi OP, Kallioniemi A, Piper J, et al. Optimizing comparative genomic hybridization for analysis of DNA sequence copy number changes in solid tumors. Genes Chromosomes Cancer 1994;10: 231-243.

36 Siproudhis L, Vilotte J, Bonfils S, et al. Idiopathic ulcerative proctitis. Clinical presentation and endoscopic outcome. Gastroenterol Clin Biol 1991;15: 315-321.

37 Ghirardi M, Nascimbeni R, Mariani PP, et al. Course and natural history of idiopathic ulcerative proctitis in adults. Ann Ital Chir 2002;73:155-159.

38 Ekbom A, Helmick C, Zack M, et al. Ulcerative colitis and colorectal cancer. A population-based study. $\mathrm{N}$ Engl J Med 1990;323:1228-1233. 